\title{
Effect of Training Program Using Functional Strength Exercises on Developing Trunk Muscles Efficiency for Mono Fin Junior Swimmers
}

\author{
Bassem Saeed Abdulazim ${ }^{1}$, Amr Albadry Mohamadine ${ }^{2}$ \\ ${ }^{1}$ Assistant Professor of Swimming at the Curricula and Teaching Methods Department, Faculty of Physical Education, Al- \\ Azhar University, Egypt \\ ${ }^{2}$ Lecturer, Faculty of Physical Education, Al-Azhar University, Egypt
}

\begin{abstract}
:
This research aimed at develop mono fin junior swimmers' physical abilities using functional strength exercises and identify their effect on following sub-aims: developing trunk (abdomen and back) muscles stability and strength for mono fin junior swimmers and improving mono fin junior swimmers' driving force in abdomen and back muscles. Ninety subjects randomly recruited from dolphin junior swimmers in Sport Police Federation Club registered in Egyptian Diving \& Lifesaving Federation in the sport season 2017 participated in the study (30 subjects in experimental group, 30 in control group and 30 for pilot study). Physical abilities tests used as data collecting tools. Experimental group undergone functional strength program consists of (44) training units, (20) training units for general preparation period, and (24) training units for specific preparation period, 5 units/week in general preparation period and 3 units/week in specific preparation period. The most important results indicated that experimental group prove superior to control group in physical variables related to mono fin swimming under study.
\end{abstract}

\section{Introduction:}

$\mathrm{M}$ ono fin swimming is one of the most popular competitions in Egyptian Diving \& Lifesaving Federation. Mono fin swimming recently developed internationally based on scientific principles, attention also given to training programs planning to improve general and private preparation especially that related to aerobic ability and muscular strength and its relationship with body composition aiming to improve mono fin swimmers technical aspects and records. (Allithy, 1990, p .232).

Swimmer performs wavy movements using trunk and legs wearing mono fin. Arms are connected and completely front elongated, head is always pointing down. If swimming on water, the swimmer breathe through an air tube. If swimming under water, an aerobic regulator placed inside the mouth so that the inhalation is through mouth and exhalation through nose (Allithy, 1990, p .234).

Abdomen muscles and its corresponding back muscles are the main physical and muscular centre for performing movement in human body. When somebody move arms and legs, he feel abdominal and back muscles moving, so he feel his physical and muscle position, which leads to easy movement control (Rateb \& Zaki, 1992, p .64)

Functional strength exercises are suitable for all ages, and sport levels. It aims to improve the relationship between muscles and nerve system. Strength and balance are of the main components of functional strength exercises. Integration between muscle force and velocity produce power, while integration between muscle force and balance produce functional strength. Functional strength exercise are important for developing abdomen and back muscles. (Boyle, 2016, p. 37 \& Metwally, 2005, p. 3)

Researchers of current study, as mono fin swimming coaches, noticed inability of some swimming juniors to maintain buoyancy status on water, and performing trunk movements from knees instead of trunk muscles. Researchers believe that this is due to insufficient trunk muscles strength (abdominal and back muscles) as well as swimmer's inability to push water effectively using trunk muscles as driving force in water.

Researchers of this study (as far as they know) noted modernity of functional strength exercise in sports field in general, and in mono fin swimming field in particular, 
although it help to strengthen trunk muscles, as strong trunk muscles strength motor conveyor from lower to upper limb. This help mono fin swimmer to swim well, because that more swimmer felt his physical status, i.e. abdomen and back muscles strength he got more confidence. These muscles as body core are responsible for balance between lower and upper limb in water, any imbalance in these areas will of course affect swimmer skill performance and record.

This research aimed at develop junior mono fin swimmers' physical abilities using functional strength exercises and identify their effect on following sub-aims:

Developing trunk (abdomen and back) muscles stability and strength for junior mono fin swimmers

Developing mono fin swimmers' driving force in abdomen and back muscles.

Researcher hypothesised the following:

There are statistically significant differences between pre and post measurements for both experimental and control groups, in trunk muscles stability and strength and some other physical variables in favour of post measurement.

There are statistically significant differences between post measurements for experimental and control groups, in variables under study in favour of experimental group.

\section{Research terms:}

Functional strength exercises: It is strength and balance exercises performed in same time (Comana, 2004, p. 27).

Trunk muscles: Abdomen and back muscles (Rateb \& Zaki, 1992, p.62).

Motor forces: The force that push swimmer's body forward, which produced through trunk and legs movements (Alkot, 2005).

Mono fin: Single fiberglass fin with two pockets where swimmer puts his feet. The foot pocket is associated with a single wide blade that provides swimmer with strength and flexibility to move feet (Metwally, 2005, p .8)

\section{Method}

\section{Subjects}

Ninety subjects randomly recruited from junior mono fin swimmers in Sport Police Federation Club registered in Egyptian Diving \& Lifesaving Federation in the sport season 2017 participated in the study (30 subjects in experimental group, 30 in control group and 30 for pilot study).

Study overall sample homogeneity confirmed as skewness factor ranged between \pm 3 , also difference between experimental and control groups were insignificant as $\mathrm{t}$ test value were insignificant at 0.05 level in main variables (Age, height, weight and training age) and physical variables (strength, flexibility and balance) under study.

\section{Measures}

1- Growth rates: Age, Height, Weight and Training age.

2- Physical tests: (appendix 2)

Researchers identified most important physical tests related to study. Physical tests enquiry form (appendix 5) presented to 10 experts (appendix 1), the physical abilities and tests obtained approval rate $80 \%$ at least accepted, and they are:

1. Right and left handgrip strength test.

2. Upper abdomen muscles strength test.

3. Lower abdomen muscles strength test.

4. Back muscles strength test.

5. Legs muscles power test.

6. Trunk flexibility test.

7. Shoulders flexibility test.

8. Backbone flexibility test.

9. Instep front and back flexibility test.

10. Static balance on instrument board

\section{Procedures}

\section{Pilot Study:}

Conducted on (30) swimmers from research community and not in main study sample in the period 8-10/1/2017 and aimed at:

1. Ensure the validity of tools and devices used in the research.

2. Work process organization and coordination, and identify how to implement the educational program.

3. Ensure assistants understand how to perform proposed exercises in training program.

4. Ensure pilot sample correct performance of some training programs units.

5. Identify the difficulties that researchers may face during study implementation.

6. Checking tests used validity and reliability.

\section{Physical and skill tests validity and reliability:}

Tests validity and reliability 
Content validity ensured by present these tests to 10 experts (appendix 1) and accept who approved all the tests with $80 \%$ approval rate at list. In addition, reliability ensured using test/retest method where correlation between the two tests ranged between 0885 and 1.000 that are significant at 0.05 level.

\section{Proposed functional strength exercises (appendix 4)}

All function strength exercises presented to experts (appendix 1) using an enquiry form (appendix 3), the following steps used while designing functional strength exercises:

\section{a-Determine proposed exercises' objective:}

The proposed exercises aimed to improve trunk muscles (abdomen and back) efficiency within junior swimmers.

b- The most important basis and principles adopted while designing functional strength exercises:

1. Exercises suitability for research sample (mono fin swimmers -15 years stage)

2. Exercises should achieve its aim (improving trunk muscles (abdomen and back)

3. Trunk strength exercises correlation with some physical variables associated with performance level (such as strength, balance, flexibility and legs muscle strength)

4. Considering good warm up to prepare muscles, and to prevent working muscles injuries.

5. Abdomen muscles training started by lower part then upper part.

6. When performing exercises from lying position, abdomen muscles should tightly contract inside, and retain the small natural gap between the back and ground.

7. When performing abdomen exercises neck lifting up should avoided, i.e. lifting upper limb instead of neck.

8. Taking into account load intensity, which exercises started with, because strong exercises burn glycogen and not fat, so researchers took care to start the proposed training program with medium intensity.

9. Moving from to another, only after all swimmers perfection of the first one.

10. Taking into account individual differences among subjects as per sports training science principles.

11. Taking into account graduation principle of exercise from easy to difficult and from simple to complex and take into account diversity principle from slow to fast.
12. The program should be flexible where some exercises can be changed.

13. Availability of security and safety factors during program implementation (Santana, 2015).

Proposed program planning and design.

\section{Proposed training program designing basis:}

- Training program should commensurate with objectives set.

- Continuity and regularity in training program practice to achieve desired benefit.

- Taking into account physiological basis of the program.

- Strength exercise should be combined with balance, stretching and relaxation exercises.

- Considering individual differences between swimmers.

- Program content diversity.

- Good warm-up and joint and backbone flexibility exercises.

- Taking into account training principles (correct timing of load repetition - Load rising gradually [graduation, developing continuity, gradual development of physical abilities])

- Continuous evaluation of motor and functional aspects to identify training effectiveness (Alkot, 2005).

\section{Program Planning:}

Based on research variables identification, selecting data collecting tools appropriate to research nature, and referring to scientific references and studies in swimming training, program implementation selected to be in general preparation period (4) weeks and specific preparation period (8) weeks totalling (12) weeks. These periods are suitable to implement the program in terms of time. Also technically, in these periods training volumes are the highest in training programs.

Numerous scientific references in sports training indicated that medium training load is between (50-74\%) and sub maximum load is between $(75-89 \%)$. The maximum load is between (90-100\%) (Albek, 2008). Researchers used training loads as following medium load (60-69\%), sub maximum load (70-79\%) and maximum load (80-90\%) as suit reteach sample in terms of their physical abilities and training age. (Figure 1)

\section{Program design:}

- The training program consists of (44) training units, (20) training units for general preparation period, and (24) 
training units for specific preparation period, 5 units/week in general preparation period and 3 units/week in specific preparation period. (appendix 7,8)

- Researchers developed muscle strength for (handgrip legs - back - abdomen) using multi - purpose weight devices.

- The proposed land training program contains the following basic elements:

- Training unit time is (45 minutes):
- (10 minutes) warmup: Warm up: Run around swimming pool, and performing general warm up exercises for all body muscles

- (30 minutes) main part: performing proposed functional strength exercises

- (5 minutes) cool down: vibration exercises to calm body and return to the normal state. (appendix 7 and 8) (Figure 1)

Figure (1)

Training load dynamics

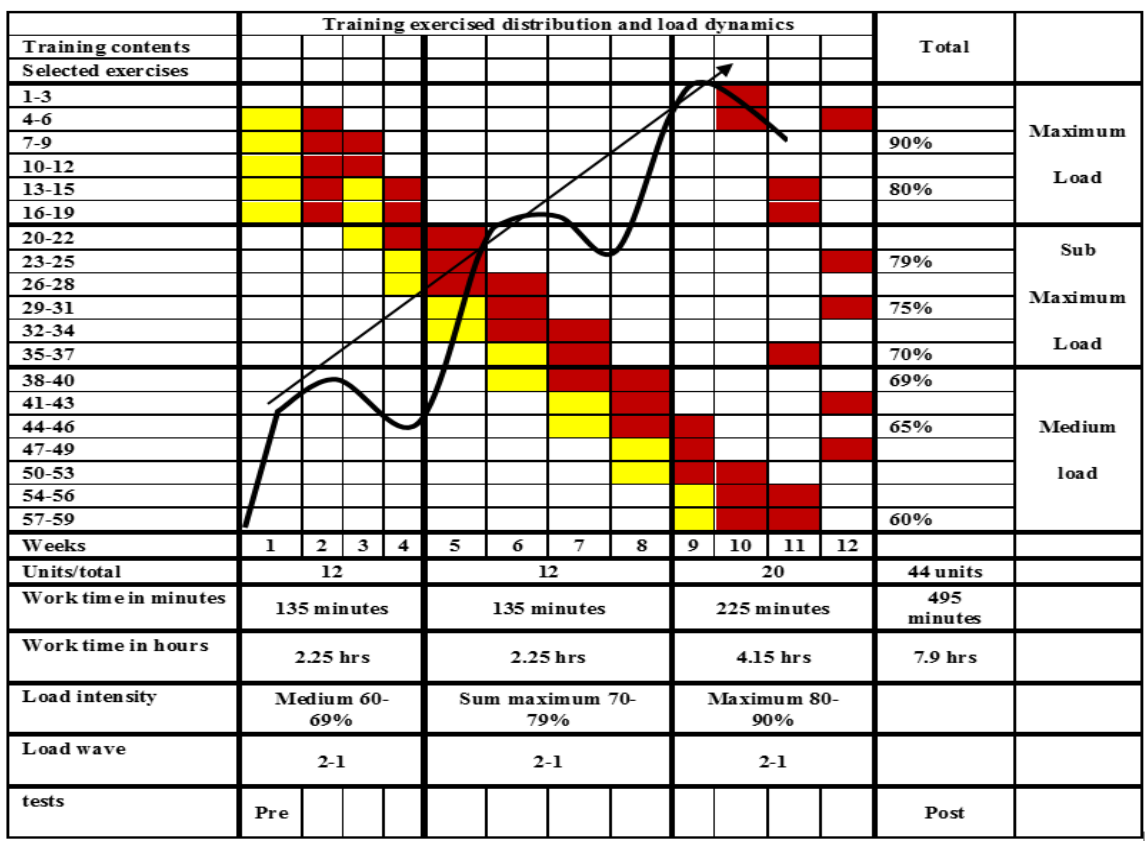

\section{Main study implementation:}

Main study implemented in the period from 15/1/2017 to 12/4/2017 as following:

\section{Pre measurement}

Pre measurement carried out for research variables on 15/1/2017 in Olympic Centre in Maadi.

\section{Main Study.}

Main study implemented in the period from Wednesday (18/1/2017) to Tuesday (11/4/2017).

\section{Post measurement}

Post measurement carried out for research variables on $12 / 4 / 2017$, with same conditions of pre measurement. Measurements recorded in predesigned form (appendix 6)
Figure 1: The dynamics of the training load

\section{Statistical process:}

SPSS statistics software program used to analyse research data. 0.05 significance level adopted throughout the research.

\section{Results}

Tables (1) and (2) demonstrate difference significance between pre and post measurements for control and experimental groups in physical variable, while table (3) demonstrates difference significance between post measurements for control and experimental groups in physical variables 
Table (1)

Difference significance between physical variables pre and post measurements for experimental group $(\mathbf{n}=\mathbf{3 0})$

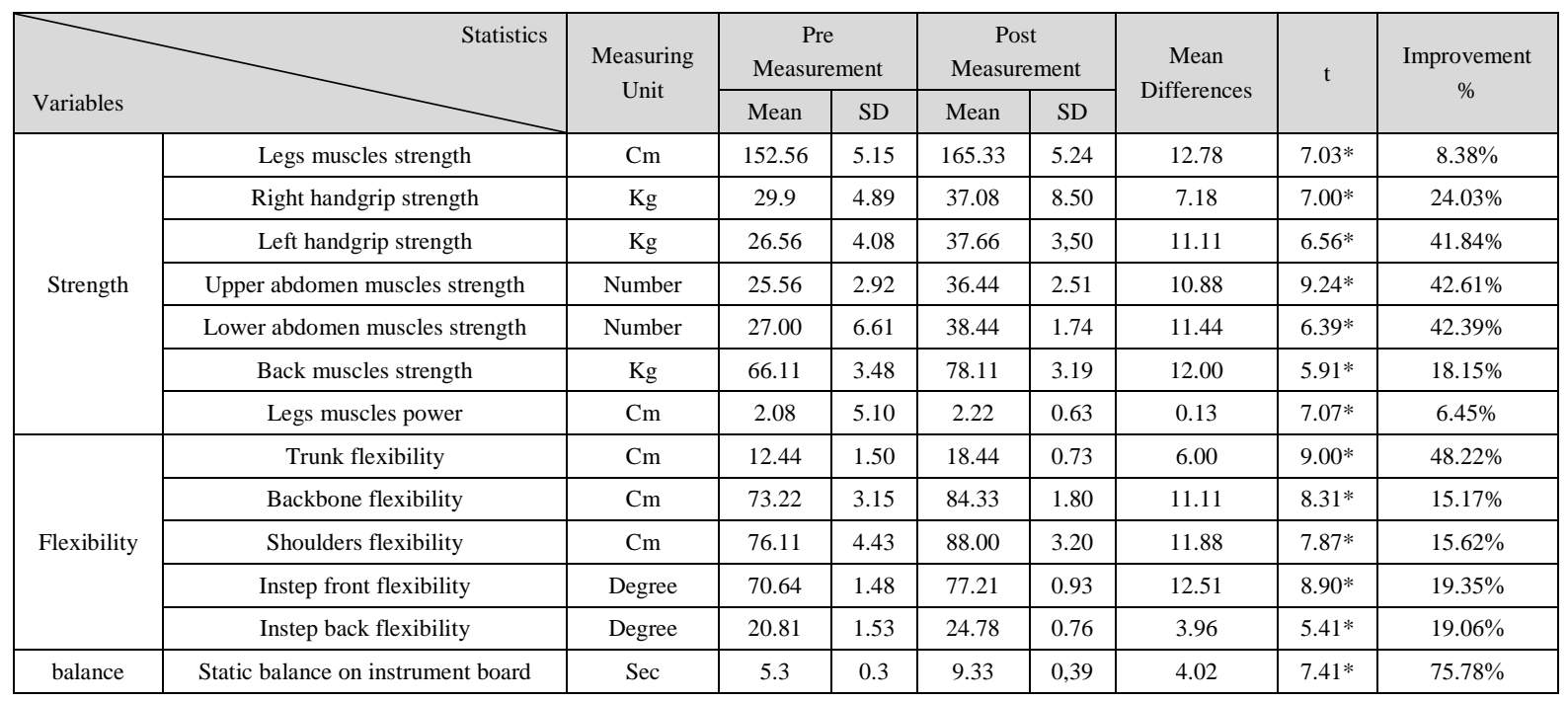

* Significant at 0.05 (t significant $=\mathbf{2 . 0 4 5})$

Table (1) results reveal statistically significant differences between experimental group's pre and post means in physical variables in favour of post measurement.

Table (2)

Difference significance between physical variables pre and post measurements for control group (n=30)

\begin{tabular}{|c|c|c|c|c|c|c|c|c|c|}
\hline \multirow{2}{*}{ Variables } & \multirow[t]{2}{*}{ Statistics } & \multirow{2}{*}{$\begin{array}{c}\text { Measuring } \\
\text { Unit }\end{array}$} & \multicolumn{2}{|c|}{$\begin{array}{c}\text { Pre } \\
\text { Measurement }\end{array}$} & \multicolumn{2}{|c|}{$\begin{array}{c}\text { Post } \\
\text { Measurement }\end{array}$} & \multirow{2}{*}{$\begin{array}{c}\text { Mean } \\
\text { Differences }\end{array}$} & \multirow[t]{2}{*}{$\mathrm{T}$} & \multirow{2}{*}{$\begin{array}{c}\text { Improvement } \\
\%\end{array}$} \\
\hline & & & Mean & SD & Mean & SD & & & \\
\hline \multirow{6}{*}{ Strength } & Legs muscles strength & $\mathrm{Cm}$ & 148.78 & 5.80 & 152.11 & 5.82 & 3.33 & $7.03 *$ & $2.24 \%$ \\
\hline & Right handgrip strength & $\mathrm{Kg}$ & 29.89 & 2.84 & 33.22 & 2.82 & 3.33 & $7.00 *$ & $11.15 \%$ \\
\hline & Left handgrip strength & $\mathrm{Kg}$ & 26.11 & 3.82 & 29,78 & 3.80 & 3.67 & $6,56^{*}$ & $14.04 \%$ \\
\hline & Lower abdomen muscles strength & Number & 26.78 & 2.11 & 29.78 & 2.59 & 3.00 & $6.39 *$ & $11.20 \%$ \\
\hline & Back muscles strength & $\mathrm{Kg}$ & 62.44 & 4.36 & 64.89 & 4.34 & 2.44 & $5.91^{*}$ & $3.92 \%$ \\
\hline & Legs muscles power & $\mathrm{Cm}$ & 2.09 & 0.49 & 2.12 & 0,50 & 0.03 & $7.07 *$ & $1.59 \%$ \\
\hline \multirow{4}{*}{ Flexibility } & Trunk flexibility & $\mathrm{Cm}$ & 13.22 & 1.09 & 14,89 & 1.36 & 1.67 & $6.00^{*}$ & $12.61 \%$ \\
\hline & Backbone flexibility & $\mathrm{Cm}$ & 72.89 & 3.02 & 75.56 & 2.96 & 2.66 & $8.32^{*}$ & $3.66 \%$ \\
\hline & Instep front flexibility & Degree & 64.36 & 1.91 & 66.26 & 1.46 & 1.89 & $8.90 *$ & $2.95 \%$ \\
\hline & Instep back flexibility & Degree & 20.05 & 1.54 & 21.88 & 1.81 & 1.82 & $5.41 *$ & $9.08 \%$ \\
\hline balance & Static balance on instrument board & $\mathrm{Sec}$ & 5.38 & 0,31 & 6.21 & $0 ., 51$ & 0.83 & $6.58 *$ & $15.41 \%$ \\
\hline
\end{tabular}

* Significant at 0.05 (t significant $=\mathbf{2 . 0 4 5})$

Table (2) results reveal statistically significant differences between control group's pre and post means in physical variables in favour of post measurement. 
Table (3)

Difference significance between post measurements for control and experimental group in physical variables $(\mathbf{n 1}=\mathbf{n} 2=30)$

\begin{tabular}{|c|c|c|c|c|c|c|c|c|c|}
\hline \multirow[b]{2}{*}{ Variables } & \multirow[t]{2}{*}{ Statistics } & \multirow{2}{*}{$\begin{array}{c}\text { Measuring } \\
\text { Unit }\end{array}$} & \multicolumn{2}{|c|}{ Experimental group } & \multicolumn{2}{|c|}{$\begin{array}{l}\text { Control } \\
\text { Group }\end{array}$} & \multirow{2}{*}{$\begin{array}{c}\text { Mean } \\
\text { Differences }\end{array}$} & \multirow{2}{*}{$\mathrm{T}$} & \multirow{2}{*}{$\begin{array}{c}\text { Effect } \\
\text { Size } \\
\text { (d) } \\
\text { Cohen }\end{array}$} \\
\hline & & & Mean & $\mathrm{SD}$ & Mean & $\mathrm{SD}$ & & & \\
\hline \multirow{7}{*}{ Strength } & Legs muscles strength & $\mathrm{Cm}$ & 165.33 & 5.24 & 152.11 & 5.82 & 13.22 & $9.246^{*}$ & 2.387 \\
\hline & Right handgrip strength & $\mathrm{Kg}$ & 37.08 & 4.89 & 33.22 & 2.82 & 3.58 & $3.745^{*}$ & 0.967 \\
\hline & Left handgrip strength & $\mathrm{Kg}$ & 37.67 & 3,500 & 29.78 & 3.80 & 7.89 & $8.365^{*}$ & 2.160 \\
\hline & Upper abdomen muscles strength & Number & 36.44 & 2.56 & 29.00 & 3.00 & 7.44 & $10.333 *$ & 2.668 \\
\hline & Lower abdomen muscles strength & Number & 38.44 & 1.74 & 29.78 & 2.59 & 8.67 & $15.202 *$ & 3.925 \\
\hline & Back muscles strength & $\mathrm{Kg}$ & 78.11 & 3.12 & 64.89 & 4.34 & 13.22 & $13.547 *$ & 3.498 \\
\hline & Legs muscles power & $\mathrm{Cm}$ & 2.22 & 0,63 & 2.12 & 0.50 & 0.9 & 0.681 & 0.176 \\
\hline \multirow{4}{*}{ Flexibility } & Trunk flexibility & $\mathrm{Cm}$ & 18.44 & 0.73 & 14.89 & 1.36 & 3.56 & $12.597 *$ & 3.253 \\
\hline & Shoulders flexibility & $\mathrm{Cm}$ & 88.00 & 3.20 & 77.78 & 4.97 & 10.22 & $9.470^{*}$ & 2.445 \\
\hline & Instep front flexibility & Degree & 77.21 & 0,93 & 66.26 & 1.46 & 10.95 & $34.647 *$ & 8.946 \\
\hline & Instep back flexibility & Degree & 24.78 & 0.76 & 21.88 & 1.81 & 2.90 & $8.091 *$ & 2.089 \\
\hline balance & Static balance on instrument board & $\mathrm{Sec}$ & 9.33 & 0.39 & 6.21 & 0,51 & 3.12 & $26.617 *$ & 6.873 \\
\hline
\end{tabular}

* Significant at $0.05(\mathrm{~T}$ significant $=\mathbf{2 . 0 0 1})$

Table (3) results reveal statistically significant differences between control and experimental groups' post means in physical variables and high effect size (>0.8) (Lakens, 2013 , p.3) (except in legs muscles power) in favour of experimental group.

\section{Discussion}

\section{First hypothesis discussion:}

\section{A - Difference significance between physical variables} pre and post measurements for experimental group

Table (1) results reveal statistically significant differences between experimental group's pre and post means in physical variables in favour of post measurement.

These results are consistent with Allithy (1990), Aldahrawy \& Ahmed (2004). Abdulmoneam (2005), Ramah \& Alhosiny (2005), Metwally (2005), and Morsi (2005) study results, which pointed out statistically significant differences between experimental groups' pre and post-measurement in some physical components as result of using training programs designed considering scientific principles and focus on functional strength training, which works in same kinetic path of different skills. These exercises work on developing special physical components and motor abilities.

Researchers attribute this experimental group's improvement to the proposed functional strength exercises programs, in this programs ground exercises used in gymnasium, playground and around the swimming pool, and focus was on various drills similar to skill performance, load intensity, volume and rest periods suits research sample. Training load components determined for each swimmer in research sample separately taking into account individual differences. Researchers took into consideration training programs designing scientific principles that characterized with speciality in developing working muscles in mono fin swimming. Researchers also considered training tools diversity according to performance requirements.

\section{B - Difference significance between physical variables pre and post measurements for control group}

Researchers return control group improvement to the traditional program, which cannot be ignored, which took into account using intensity gradual resistances aiming to improve physical variables and make swimmers access best possible performance. Traditional program also included developing physical fitness elements in general without addressing attention to certain body part, the matter that resulted in low improvement percentage for control group.

Thus, first hypothesis accepted.

\section{Second hypothesis discussion:}

Table (3) results reveal statistically significant differences between control and experimental groups' post means and 
high effect size in physical variables in favour of experimental group.

These results are consistent with Metwally (2002) study results, which pointed out statistically significant differences between post-measurements for experimental and control groups in physical variables as result of using standardized training programs that designed considering scientific principles and suitable times for mono fin swimmers. Training load standardized in terms of intensity and volume that led to developing research sample swimmers' physical variables. Using functional strength exercises, tools diversity, and exercised suitability to research sample's age led to experimental group improving superiority compared with control group.

Researchers attribute these results to the fact that functional strength training deals with body movement functions and indispensable abilities for success in competition, improving sports performance and enhancing it through advanced exercises that stimulate specific patterns of common movements used in sports. These exercises perfection lead player to high degree of stability, balance and strength in performance and this is the aim of functional strength exercises and programs.

In this regard, Cress, Conley, Balding, Hansen-Smith, \& Konczak (1996) argues that functional strength exercises are suitable for all ages at training levels and aims to improve the relationship between muscles and nervous system, and adds that strength and balance are key elements of functional strength exercises. Muscle strength and motor speed integration result in muscle strength or power, while integration of muscle strength and balance results in functional strength.

Morsi (2005) suggests that the primary aim of functional strength programs is to increase muscular strength, protect muscles, achieve self-stability and neuromuscular control in trunk muscles, and also produce strength and convert it to immediate speed.

Reginato, Cymara, McGibbon, Scarborough, \& Krebs (2005) in the opinion that all training programs should include functional strength exercises. They proof their opinion with the fact that all players during competition in different sporting activities not evenly based on both feet and on one line except for periods like rowing. Therefore, the exercises that practiced mostly from sitting or standing position are not suitable for athletes in most sports activities.

Muir, MacGregor, McCann, \& Hillis (1999), Ekstrom, Donatelli, \& Carp (2007), Navalta \& Hrncir (2007), Desai
\& Marshall (2010), and Desai \& Marshall(2011) studies pointed out importance of training in strengthening abdominal and back muscles which lead to developing and improving abdomen and back muscular performance and in turn raise motor performance level.

Thus, second hypothesis accepted.

\section{Conclusions}

1- Experimental group improvement percentage higher than control group because of exercises used in proposed program

2- Proposed functional strength exercises effectively contributed to developing trunk muscles (abdomen and back muscles) as body centre.

3- Using several tools in exercises performance led muscle strength improvement, which in turn increased balance when performing mono fin swimming.

4- Functional strength exercises improved main muscles, which in turn led to strength absorption and transfer.

5- The results showed that the strength of the functional strength of the research sample was appropriate for the age stage under study.

\section{Recommendations}

1- Using proposed functional strength exercises in general and specific preparation period to reduce some problems faced by swimmers, such as weakness of frontal abdominal muscles, strength and shortness of trunk back muscles, which requires the strengthening abdominal muscles and stretching back muscles, which lead to mono fin swimmers' records.

2- Giving attention to abdomen and back strength exercises in warm-up, which help swimmers to improve physical performance level.

3- Conduct more studies on most important exercises that contribute to abdomen and back muscles' strength development for different age stages and best ways to develop it.

4- Take into account selected exercises similarity with mono fin swimming performance or similarity to muscles' work under water.

5- Draw coaches' attention to take into account standardising training loads for mono fin swimmers individually and, and considering training physiological basis and principles. 
6- Conduct similar studies to identify working muscles in mono fin swimming and its participation proportion.

7- Conduct similar studies on dual fins swimming to upgrade physical level of dual fins swimmers.

\section{References}

1- Abdulmoneam, D. M. (2005). Effect of suggested program for abdomen and back muscles on some body composition variables and sudden and strong movements' performance level in modern dance (Unpublished master's thesis). Faculty of physical Education for girls, Zagazig University, Zagazig, Egypt. (In Arabic)

2- Albek, A. (2008). Modern trends in sports training. Alexandria, Egypt: Al-Maaref establishmenr.

3- Aldahrawy, M. M., \& Ahmed, M. M. (2004). Effectiveness of integrative functional exercises on some physical variables and major blood elements level and short distances swimming records. Zagazig University Medical Journal, (1), 1-27. (In Arabic)

4- Alkot, M. (2005). Swimming sports training strategy. Cairo, Egypt: Book publishing center.

5- Allithy, S. H. (1990). Relation between lower limb strength and flexibility and legs strocks in front crawl swimming. Faculty of physical Education, Helwn University journal, (26). (In Arabic)

6- Boyle, M. (2016). New functional training for sports. Champaign, IL: Human Kinetics.

7- Comana, F. (2004). Function training for sports. Champaign, IL: Human Kinetics.

8- Cress, M. E., Conley, K. E., Balding, S. L., HansenSmith, F., \& Konczak, J. (1996). Functional training: Muscle structure, function, and performance in older women. Journal of Orthopaedic \& Sports Physical Therapy, 24(1), 4-10. doi:10.2519/jospt.1996.24.1.4

9- Desai, I., \& Marshall, P. W. (2010). Acute effect of labile surfaces during core stability exercises in people with and without low back pain. Journal of Electromyography and Kinesiology, 20(6), 1155-1162. doi:10.1016/j.jelekin.2010.08.003

10- Ekstrom, R. A., Donatelli, R. A., \& Carp, K. C. (2007). Electromyographic analysis of core trunk, hip, and thigh muscles during 9 rehabilitation exercises. Journal of Orthopaedic \& Sports Physical Therapy, 37(12), 754-762. doi:10.2519/jospt.2007.2471
11- Lakens, D. (2013). Calculating and reporting effect sizes to facilitate cumulative science: a practical primer for t-tests and Anovas. Frontiers in Psychology, 4, 1-12.

12- Metwally, M. M. (2005). Effectiveness of functional strength training on some physical variables, skill variables, and major blood elements level within football buds. Egyptian Journal of Basic and Applied Physiology, 5(2). (In Arabic)

13- Metwaly, D. A. (2002). Effect of training program using mono fin on legs flexibility and muscular strength and their relation to swimmers' records (Unpublished master's thesis). Faculty of physical Education for girls, Zagazig University, Zagazig, Egypt. (In Arabic)

14- Morsi, A. M. (2005). Effectiveness of integrative functional exercises on some physical variables, balances, and turns skill performance in rhythmic exercises. Integrated physical education researches, Faculty of physical Education for girls, Zagazig University, (5). (In Arabic)

15- Muir, D. F., MacGregor, G. D., McCann, G. P., \& Hillis, W. (1999). The prevalence of left ventricular hypertrophy in elite professional footballers. International Journal of Cardiology,71(2), 129-134. doi:10.1016/s0167-5273(99)00133-3

16- Navalta, J. W., \& Hrncir, S. P. (2007). Core stabilization exercises enhance lactate clearance following high-intensity exercise. The Journal of Strength and Conditioning Research,21(4), 1305. doi:10.1519/r21546.1

17- Ramah, N. H., \& Alhosiny, N. M. (2005). Effectiveness of integrative functional exercises on some physical variables and gymnastics movements performance level. Integrated physical education researches, Faculty of physical Education for girls, Zagazig University, (4). (In Arabic)

18- Rateb, O. K., \& Zaki, A. M. (1992). Scientific bases of swimming training. Cairo, Egypt: Dar Al-Fekr Al-Arabi. (In Arabic)

19- Reginato, A. M., Cymara, C. P., McGibbon, C. A., Scarborough, D. M., \& Krebs, D. A. (2005). Chair rise and lifting characteristics of elders with knee osteoarthritis (OA): Functional and strengthening effects. Osteoarthritis and Cartilage, 13, S33. doi:10.1016/s10634584(05)80395-4

20- Santana, J. C. (2015). Functional training. Chmpaign, IL: Human Kinetics. 
21- Sharrock, C., Cropper, J., Mostad, J., Johnson, M., \& athletic performance: is there a relationship? International Malone, T. (2011). A pilot study of core stability and Journal of Sports Physical Therapy, 6(2), 63-74. 